\title{
Actinobacterial Biofertilizers: An Alternative Strategy for Plant Growth Promotion
}

\author{
Kavita Rani*, Anupma Dahiya, Jeniffer Christeena Masih and Leela Wati \\ Department of Microbiology, Chaudhary Charan Singh Haryana Agricultural University, \\ Hisar, Haryana-125004, India \\ *Corresponding author
}

\section{A B S T R A C T}

The problem of food security arises along with the increase in world population. To meet the enormous food demands of growing population, farmers use traditional agricultural practices which mainly rely on use of chemical fertilizers

\section{Keywords}

Plant growth

Promotion,

Actinobacterial

biofertilizers

Article Info

Accepted:

06 August 2018

Available Online:

10 September 2018 and pesticides which are extensively harmful to the humans as well as environment. Therefore, there is an immense demand for an alternate strategy to increase the food productivity and quality which does not rely on use of these harmful chemicals. This increasing demand of lesser use of these chemicals has led to the use of soil microorganisms which possess the ability of nutrient cycling, improve soil quality and plant health as well as crop productivity. Among soil microorganisms, actino-bacteria represent an important group of microorganisms which has been reported to produce some useful substances which help in increasing soil quality and improve plant growth promotion as well as crop productivity. Hence, actino-bacteria represent a key component of agricultural ecosystems. This is important to increase our knowledge about interaction of these microorganisms with the soil ecosystem. On the basis of several studies, the present article highlights the importance of actino-bacteria in plant growth promotion by various means

\section{Introduction}

Rhizosphere is an area where a strong microbiological bustle takes place due to the release of various kind of plant metabolites, known as root exudates. These root exudates consist of various amino acids, sugars, fatty acids, proteins, vitamins, etc. which play an important role to dwell microorganisms in the rhizosphere. A vast group of microorganisms inhabit the rhizosphere and show ecological activities by interacting with plants and other microorganisms. Actinobacteria comprise a major group of microorganisms which is found in rhizosphere as well as inside the plant roots as endophytes (Bhosale and Kadam, 2015). Although, the population of actinobacteria in the rhizosphere is different 
from the endophytic environment due to the presence of root exudates and other microorganisms in the rhizosphere, both kinds of communities use related mechanisms to promote the plant growth. Actinobacteria are well known for their productive activities in nutrient recycling by degradation of chitin, cellulose, starch, lipids and complex carbohydrates and flouting them into simple sugars by the secretion of various kinds of hydrolytic enzymes in the rhizosphere (Vurukonda et al., 2018). These microorganisms, when, reside around the plant root surfaces, perform an important role in breakdown of organic matter and make it available for the plant uptake. These microorganisms also show their potential role in solubilization of rock phosphate, production of siderophores, indole-acetic-acid (IAA), hydrogen cyanide (HCN), ammonia and lytic enzymes (Jog et al., 2012; Damam et al., 2016). Actinobacteria may also suppress the venomous microorganisms which could be responsible for inhibition of plant growth. These abilities of actinobacteria have positive effects on the plant growth promotion and thereby, actinobacteria are also known as "plant growth promoting rhizobacteria".

Sustainable agriculture is a fundamental need of today's world because it is the only mean to ensure food security and food quality. It has the potential to meet our imminent requirements of agricultural products. Traditional agricultural practices, which include the use of harmful chemical fertilizers and pesticides, will not be able to meet these colossal agricultural needs. To retain sustainable agriculture, it is essential to limit the use of these harmful chemicals and replace them with ecofriendly agricultural practices. Plant growth promoting actinobacteria, also known as biofertilizers, being environment friendly and renewable, offer superior alternative to these hazardous and non-renewable fertilizers and pesticides. Actinobacteria promote the plant growth by two possible mechanisms i.e. direct and indirect mechanisms (Aditi and Anupama, 2015). These mechanisms involve nitrogen fixation, phosphate solubilization, production of phytohormone such as indole-acetic acid (IAA), utilization of 1- aminocyclopropane-1carboxylate (ACC), production of siderophores, cyanide $(\mathrm{HCN})$, lytic enzymes and antibiotics. These mechanisms are either engaged in plant growth promotion directly by supplying nutrients to the plants or suppress the deleterious microorganisms dwelling around the plant roots which could be harmful to the plant growth. Therefore, these direct and indirect plant growth promoting characteristics of actinobacteria make them superior alternative to the hazardous chemicals.

\section{Nitrogen fixation}

Nitrogen is a very critical element for limiting the growth of plants due to its unavailability to the plant uptake. It is a vital component of the most needed pigment for photosynthesis i.e. chlorophyll, in addition to amino-acids, proteins, ATP molecules and nucleic acids. Nitrogen is the most abundant element on earth's atmosphere in its molecular form $\left(\mathrm{N}_{2}\right)$ (Vance, 2001). However, plants can use only reduced form of nitrogen as either ammonium $\left(\mathrm{NH}_{4}{ }^{+}\right)$or nitrate $\left(\mathrm{NO}_{3}{ }^{-}\right)$. The molecular nitrogen is generally reduced via physical, chemical and biological means. Over physical and chemical means of nitrogen fixation, biological nitrogen fixation is an efficient method to fix the molecular nitrogen into its reduced forms which are more readily taken up by the plants. Nitrogen fixation via biological means entails the role of microorganisms. Among actinobacteria, Frankia is a resourceful microorganism which can fix the molecular nitrogen in nonleguminous actinorhizal plants under 
symbiotic as well as free living conditions (Sathya et al., 2017). It infects the actinorhizal plant roots either by forming a thread like structure intracellularly or by intercellular cell incursion. Frankia form vesicles in the roots of the actinorhizal plants under $\mathrm{N}$ deficient and normal oxygen tension conditions where nitrogen fixing enzyme, nitrogenase, carry out the fixation of molecular nitrogen.

Beside Frankia, non-Frankia actinomycetes have also been found to fix the nitrogen under non-symbiotic conditions. An unusual group of actinomycetes showing positive acetylenereduction activity has been illustrated in literature which was retrieved from surfacesterilized roots of Casuarina equisetifolia growing in Mexico and their distinction from Frankia was confirmed using 16S rRNA gene phylogenetic analysis and DNA-DNA homology which was found very low with Frankia (Valdes et al., 2005). These nonFrankia actinomycetes, found to be closely related to Thermomonosporaceae and the Micromonosporaceae, were not only able to grow on $\mathrm{N}$-free medium and found positive to acetylene-reduction activity but also found to possess nifH gene responsible for $\mathrm{N}$-fixation. Therefore, the potential of some special actinobacteria to fix atmospheric nitrogen plays a significant role to supply nutrients for the growth of plants.

\section{Phosphate solubilization}

Like nitrogen, phosphorous is another essential element for all living life on the earth and in soil environment, it is an important limiting factor for plant growth. Soil phosphorous occurs in organic as well as in inorganic forms. The amount of organic and inorganic phosphorous in soil depends largely on soil properties, like $\mathrm{pH}$ and type of soil. Organic phosphorous accounts for 29 $65 \%$ of total soil $\mathrm{P}$ content, but in some soil types, it contributes upto $90 \%$ total soil $\mathrm{P}$ (Ghorbani-Nasrabadi et al., 2013). Usually, $\mathrm{P}$ content in the soil is present in excess for the use of plants. Nonetheless, a very little fraction of this huge amount of $\mathrm{P}$ in soil is readily available to the plants because $P$, being highly reactive, makes complexes with other elements which are not taken up by the plants. Plants can uptake only monobasic and dibasic form of $\mathrm{P}$. To fulfill the requirements of $\mathrm{P}$, traditional agricultural practices make use of rock phosphate fertilizers which result in depletion of phosphate reservoirs.

Actinobacteria are prime microorganisms in the soil representing crucial role in nutrient recycling. They have recently been reported to solubilize complexes of phosphate and making $\mathrm{P}$ available to the plants uptake. These microorganisms have the potential to carry out hydrolysis of phytate which is the most prevalent form of organic (inositol) phosphate in soil.

Actinomycetes Streptomyces alboniger, $S$. venezuelae, $S$. ambofaciens and $S$. lienomycini have been demonstrated to produce extracellular phytate-degrading enzymes i.e. phytases which correspond to a group of phosphomonoesterases that commence the stepwise breakdown of phytate (GhorbaniNasrabadi et al., 2012). On the basis of $\mathrm{pH}$, these phytases have been recorded as acid and alkaline phytases.

Another mean of solubilization of phosphate by soil microflora is the production of various acids i.e. gluconic acid, citric acid, malic acid, succinic acid and oxalic acid which depends on metabolic pathways to utilize different carbon sources. However, mechanism of acidification in phosphate solubilization by actinobacteria is rarely reported (Jog et al., 2014). Therefore, enzymatic degradation of phosphate complexes plays a critical role in making $\mathrm{P}$ available to the plants. The ability 
of actinobacteria to solubilize $\mathrm{P}$ makes them a better candidate to use as natural fertilizers.

\section{Production of phytohormone}

Root exudates secreted by the plants in rhizosphere help to modulate the microflora around the roots of plants and construct potential environment for the synthesis of IAA by the microorganisms dwelling in the rhizosphere. Actinobacteria have been studied to produce phytohormone belonging to class of auxins i.e. IAA which is a common plant hormone (Kamal et al., 2014). Indole-acetic acid production helps in growth and development of plants by promoting cell division and elongation. Among actinobacteria, the production of IAA (71 $\mathrm{g} / \mathrm{mL}$ and $197 \mathrm{~g} / \mathrm{mL}$ ) by two different Streptomyces sp. have been revealed to enhance seed germination and seedling growth of a folk ethno-medicinal plant of Meghalaya, Centella asiatica (Dochhil et al., 2013). The production of IAA has also been mentioned in other Streptomyces sp. also, including Streptomyces violaceus, Streptomyces griseus, Streptomyces exfoliates, Streptomyces coelicolor, Streptomyces lividans etc. (Vurukonda et al., 2018). Their ability to produce IAA makes them a potential candidate for use in agricultural practices as natural fertilizers to maintain sustainability of agricultural products.

\section{Utilization of 1- aminocyclopropane-1- carboxylate (ACC)}

Some actinobacteria have the ability to use ACC which acts as a precursor molecule for the biosynthesis of ethylene in the plants. Ethylene is often called as 'aging hormone' because of its role in enhancing plant developmental processes which include ripening, senescence and abscission (Schaller, 2012). An enzyme, ACC-deaminase catalyzes the hydrolysis of ACC into ammonia and alpha-ketoglutarate. Among actinobacteria, streptomycetes have been evaluated to produce ACC-deaminase (El-Tarabily, 2008). Their study revealed increased plant growth promotion of tomato (Lycopersicon esculentum Mill.) by Streptomyces filipinensis and $S$. atrovirens due to the production of ACC-deaminase. Furthermore, S. filipinensis has been reported to promote plant growth more as compared to $S$. atrovirens due to the production of IAA and ACC-deaminase both by $S$. filipinensis while $S$. atrovirens has been reported to produce ACC-deaminase only. Therefore, it is deemed that actinobacteria showing more plant growth promoting properties are more prominent to use as biofertilizers.

\section{Production of siderophores}

Iron is a very essential element in all the living organisms as it plays an important role in catalysis of numerous enzymatic reactions where it acts as a co-factor. Earlier, iron was usually present in ferrous form $\left(\mathrm{Fe}^{2+}\right)$ in soil during oxygen deficient atmosphere, which was easily utilized by the microorganisms. However, with the passage of time, as the oxygen deficient atmosphere replaced by oxygen rich environment, iron get oxidized to ferric form $\left(\mathrm{Fe}^{3+}\right)$ which is not readily utilized by microorganisms. To overcome this challenge, microorganisms evolved to produce small, low molecular weight, iron chelating molecules i.e. siderophores which form complexes with iron (Wilson et al., 2016). The competition for iron acquisition occurs between plants and phytopathogens as microbial siderophores have higher affinity towards iron chelation making it unavailable to the plants.

Streptomyces sp. have been reported to produce siderophores i.e. 'coelichelin', a peptide siderophores by Streptomyces 
coelicolor (Challis and Ravel, 2000), 'enterobactin' by $S$. tendae and Streptomycin sp. Tu 6125 (Fiedler et al., 2001). Siderophore producing actinobacteria create iron deficient conditions for phytopathogens by chelating the iron present in the rhizosphere and help to protect plants from disease which leads to the better growth of plants.

\section{Production of cyanide}

Actinobacteria have the ability to produce hydrogen cyanide ( $\mathrm{HCN})$. The mechanism of action of $\mathrm{HCN}$ is considered to inhibit terminal 'cytochrome c oxidase' in the respiratory chain and binds to metalloenzymes which confers it the property of suppressing phytophathogens (Ramette et al., 2003; Olanrewaju et al., 2017). Different species of Streptomyces have been reported to produce $\mathrm{HCN}$ conferring important role in disease suppression (Passari et al., 2015; Anwar et al., 2016). Hydrogen cyanide has also been reported to contribute in mineral mobilization and phosphate release which results in indirect increase of nutrient availability to both actinobacteria and their host plants (Rijavec and Lapanje, 2016). Based on the ability of $\mathrm{HCN}$ to prevent plant pathogen and to enhance nutrient availability, $\mathrm{HCN}$ producing actinobacteria can be used as biocontrol as well as plant growth promoting agents.

\section{Production of lytic enzymes}

Cell wall of any organism is accountable to maintain the integrity of cells under all kinds of environment i.e. isotonic, hypotonic and hypertonic. Cell wall of different organisms is composed of various kinds of complex polymeric substances, for instance, fungal cell wall is composed of chitin and $\beta-1,3$-glucan while cell wall of oomycetes is mainly composed of cellulose and $\beta-1,3$-glucan.
Furthermore, bacterial cell wall is composed of peptidoglycan i.e. polysaccharide chain cross linked with unusual peptides. Actinobacteria have been observed to produce various lytic enzymes which hydrolyze the cell wall component of other bacteria, fungi and protozoa and thus, prevent harmful microorganisms to cause disease. Streptomyces albovinaceus, $S$. caviscabies, $S$. griseus, $S$. setonii and $S$. virginiae have been reported to produce chitinases (Macagnan et al., 2008). Streptomyces RC1071 retrieved from cerrado soil was tested against phytopathogenic fungus which was observed having antifungal activity (Gomes et al., 2001). Actinomycetes have also been illustrated to produce proteases, lipases and cellulases (Aditi and Anupma, 2015). The production of lytic enzymes by actinobacteria grants them biocontrol potential and aids the plant growth promoting characteristics.

\section{Production of antibiotics}

Actinobacteria have been extensively studied to produce a vast variety of secondary metabolites i.e. by-products of metabolism which are not generally essential for their own growth (Waksman et al., 2010; Nanjwade et al., 2010; Omran and Kadhem, 2016). These secondary metabolites are termed as 'antibiotics'. Antibiotics exhibit antitumoral (doxorubicin and bleomycin), antifungal (amphotericin B and nystatin), immunosuppressive (FK-506 and rapamycin), insecticidal (spinosyn A and avermectin B), herbicidal (phosphinotricin) and many clinically and commercially important activities (Grasso et al., 2016). Most of the antibiotics with diverse biological activities are produced by actinomycetes. Among actinomycetes, Streptomyces sp. have been reported to produce a wide variety of antibiotics belonging to class $\beta$-lactam (Ram, 2014). Antibiotics differ in their chemical structure, mode of action and effects on 
different organisms. In rhizosphere, antibiotics produced by soil dwelling actinomycetes play a very significant role in inhibiting the growth of plant pathogens by targeting either essential molecules or biosynthetic pathways. Production of antibiotics depends upon several environmental factors, such as temperature, $\mathrm{pH}$, aeration, presence of competitor microorganisms etc. (Omran and Kadhem, 2016). Therefore, actinobacteria present in soil produce a variety of antibiotics depending upon environmental conditions and these antibiotics inhibit a wide range of pathogenic microorganisms to cause disease in the plants. As a result, actinobacteria aid to the better plant health and development leading to the sustainability of agricultural products.

\section{Conclusion and future prospectives}

Actinobacteria possess a great potential to enhance plant growth and development by producing various substances which increase nutrient supply to the plants, provide essential phytohormones, inhibit the growth of harmful microorganisms in rhizosphere and suppress disease to occur. These abilities of actinobacteria make them a competent candidate to use as biofertilizers and biocontrol agents to attain sustainability in agriculture. Use of these plant growth promoting actinobacteria helps to limit the use of chemical fertilizers and pesticides which could either harm the environment and devastate agricultural sustainability to a very large extent.

Some actinobacteria are having a few while the other possesses several plant growth promoting characteristics. This can limit their use to attain sustainability in agriculture because a microorganism with maximum number of plant growth promoting characteristics is considered as an ideal candidate for use. Therefore, genetic manipulations of an optimal actinobacterial candidate to the better one are needed to be accepted upto safe and sound levels by the scientists, breeders and regulatory agencies to achieve a very giant goal of increased crop productivity without environmental hazards. Therefore, a lot of work is to be done genetically to improve the efficacy of actinobacteria in plant growth promotion and suppression of diseases by bioactive compounds.

\section{References}

Aditi, S. and Anupama, T. 2015. Symbiotic organisms: key for plant growth promotion. International Journal of Science, Engineering and Technology Research. 4(4): 1108-1113.

Anwar, S., Ali, B. and Sajid, I. 2016. Screening of rhizospheric actinomycetes for various in-vitro and in-vivo plant growth promoting (PGP) traits and for agroactive compounds. Frontiers in Microbiology. 7(1334): 111.

Bhosale, H. J. and Kadam, T. A. 2015. Generic diversity and a comparative account on plant growth promoting characteristics of actinomycetes in roots and rhizosphere of Saccharum officinarum. International Journal of Current Microbiology and Applied Sciences. 4(1): 230-244.

Challis, G. L., and Ravel, J. 2000. Coelichelin, a new peptide siderophore encoded by the Streptomyces coelicolor genome: structure prediction from the sequence of its non-ribosomal peptide synthetase. FEMS Microbiology Letters. 187(2): 111-114.

Damam M., Moinuddin, M. K. and Kausar R. 2016. Isolation and screening of plant growth promoting actinomycetes from rhizosphere of some forest medicinal 
plants. International Journal of ChemTech Research. 9(5): 521-528.

Dochhil, H., Dkhar, M. S. and Barman, D. 2013. Seed germination enhancing activity of endophytic Streptomyces isolated from indigenous ethnomedicinal plant Centella asiatica. International Journal of Pharma and Bio Sciences. 4(1): $256-262$.

El-Tarabily, K. A. 2008. Promotion of tomato (Lycopersicon esculentum Mill.) plant growth by rhizosphere competent 1aminocyclopropane-1- carboxylic acid deaminase-producing streptomycete actinomycetes. Plant Soil. DOI 10.1007/s11104-008-9616-2.

Fiedler, H., Krastel, P., Muller, J., Gebhardt, K. and Zeeck, A. 2001. Enterobactin: the characteristic catecholate siderophores of enterobacteriaceae is produced by Streptomyces species. FEMS Microbiology Letters. 196: 147151.

Ghorbani-Nasrabadi, R., Greiner, R., Alikhani, H. A. and Hamedi, J. 2012. Identification and determination of extracellular phytate-degrading activity in actinomycetes. World Journal of Microbiology and Biotechnology. 28: 2601-2608.

Ghorbani-Nasrabadi, R., Greiner, R., Alikhani, H. A., Hamedi, J. and Yakhchali, B. 2013. Distribution of actinomycetes in different soil ecosystems and effect of media composition on extracellular phosphatase activity. Journal of Soil Science and Plant Nutrition. 13(1): 223236.

Gomes, R. C., Semedo, L. T. A. S., Soares, R. M. A., Linhares, L. F., Ulhoa, C. J., Alviano, C. S. and Coelho, R. R. R. 2001. Purification of a thermostable endochitinase from Streptomyces RC1071 isolated from a cerrado soil and its antagonism against phytopathogenic fungi. Journal of Applied Microbiology. 90: 653-661.

Grasso, L. L., Martino, D. C. and Alduina, R. 2016. Production of antibacterial compounds from actinomycetes. In: Dhanasekaran D, Jiang Y (Eds.) Actinobacteria, Basics and Biotechnological Applications. Intech Open Access Publication, pp. 177-198.

Jog, R., Nareshkumar, G. and Rajkumar, S. 2012. Plant growth promoting potential and soil enzyme production of the most abundant Streptomyces spp. from wheat rhizosphere. Journal of Applied Microbiology. 113, 1154-1164.

Jog, R., Pandya, M., Nareshkumar, G. and Rajkumar, S. 2014. Mechanism of phosphate solubilization and antifungal activity of Streptomyces spp. isolated from wheat roots and rhizosphere and their application in improving plant growth. Microbiology. 160: 778-788.

Kamal, R., Gusain, Y. S. and Kumar, V. 2014. Interaction and symbiosis of AM fungi, actinomycetes and plant growth promoting rhizobacteria with plants: strategies for the improvement of plants health and defense system. International Journal of Current Microbiology and Applied Sciences. 3(7): 564-585.

Macagnan, D., Romeiro, R. S., Pomella, A. W.V. and Souza, J. T. 2008. Production of lytic enzymes and siderophores, and inhibition of germination of basidiospores of Moniliophthora (ex Crinipellis) perniciosa by phylloplane actinomycetes. Biological Control. 47: 309-314.

Nanjwade, B. K., Chandrashekhara, S., Goudanavar, P. S., Shamarez, A. M. and Manvi, F. V. 2010. Production of antibiotics from soil-isolated actinomycetes and evaluation of their antimicrobial activities. Tropical Journal of Pharmaceutical Research. 9 (4): 373-377. 
Olanrewaju, O. S., Glick, B. R. and Babalola, O. O. 2017. Mechanisms of action of plant growth promoting bacteria. World Journal of Microbiology and Biotechnology. 33(197): 1-16.

Omran, R. and Kadhem, M. F. 2016. Production, purification and characterization of bioactive metabolites produced from rare actinobacteria Pseudonocardia alni. Asian Journal of Pharmaceutical and Clinical Research. 9(3): 264-272.

Passari, A. K., Mishra, V. K., Gupta, V. K., Yadav, M. K., Saikia, R. and Singh, B. $\mathrm{P}$. 2015. In vitro and in vivo plant growth promoting activities and DNA fingerprinting of antagonistic endophytic actinomycetes associates with medicinal plants. PLOS ONE. 118.

Ram, L. 2014. Optimization of medium for the production of streptomycin by Streptomyces griseus. International Journal of Pharmaceutical Science Invention. 3(11): 1-8.

Ramette, A., Frapolli, M., Défago, G. and Moënne-Loccoz, Y. 2003. Phylogeny of $\mathrm{HCN}$ synthase-encoding hcnBC genes in biocontrol fluorescent pseudomonads and its relationship with host plant species and HCN synthesis ability. Molecular Plant-Microbe Interactions. 16(6): 525-535.

Rijavec, T. and Lapanje, A. 2016. Hydrogen cyanide in the rhizosphere: not suppressing plant pathogens, but rather regulating availability of phosphate. Frontiers in Microbiology. 7(1785): 111.
Sathya, A., Vijayabharathi, R. and Gopalakrishnan, S. 2017. Plant growthpromoting actinobacteria: a new strategy for enhancing sustainable production and protection of grain legumes. 3 Biotech. 7: 1-10.

Schaller, G. E. 2012. Ethylene and the regulation of plant development. BMC Biology. 10(9): 1-3.

Valdes, M., Perez, N. O., Santos, P. E., Caballero-Mellado, J., Pena-Cabriales, J. J., Normand, P. and Hirsch, A. M. 2005. Non-Frankia actinomycetes isolated from surface-sterilized roots of Casuarina equisetifolia fix nitrogen. Applied and Environmental Microbiology. 71(1): 460-466.

Vance, C. P. 2001. Symbiotic nitrogen fixation and phosphorus acquisition; plant nutrition in a world of declining renewable resources. Plant Physiology. 127: 390-397.

Vurukonda, S. S. K. P., Giovanardi, D. and Stefani, E. 2018. Plant growth promoting and biocontrol activity of Streptomyces spp. as endophytes. International Journal of Molecular Sciences. 19: 1-26.

Waksman, S. A., Schatz, A. and Reynolds, D. M. 2010. Production of antibiotic substances by actinomycetes. Annals of the New York Academy of Sciences. 1213: 112-124.

Wilson, B. R., Bogdan, A. R., Miyazawa, M., Hashimoto, K. and Tsuji, Y. 2016. Siderophores in iron metabolism: from mechanism to therapy potential. Trends in Molecular Medicine. 22(12): 10771090.

\section{How to cite this article:}

Kavita Rani, Anupma Dahiya, Jeniffer Christeena Masih and Leela Wati. 2018. Actinobacterial Biofertilizers: An Alternative Strategy for Plant Growth Promotion. Int.J.Curr.Microbiol.App.Sci. 7(09): 607-614. doi: https://doi.org/10.20546/ijcmas.2018.709.072 